\title{
Enhanced attitude control structure for small satellites with reaction wheels
}

\begin{abstract}
Purpose: This paper aims to describe a design enhancement for the satellite attitude control system using reaction wheels, and the wheel momentum unloading using magnetorquers. Design/methodology/approach: The proportional - integralderivative-controller and active force control (AFC) schemes are developed together with their governing equations for closed loop system of attitude control. Four numerical simulations were carried out using the Matlab - Simulink ${ }^{\mathrm{TM}}$ software and results were compared. Findings: From the results, it is evident that the attitude accuracies for roll-pitch-yaw axes have improved significantly through the proportional - derivative (PD) - AFC controller for the attitude control and the wheel momentum can be well maintained during the momentum unloading scheme. The results show that the AFC has a high potential to be implemented in the satellite attitude control system. Practical implications: Using AFC, the actual disturbance torque is considered totally rejected by the system without having to have any direct prior knowledge on the actual disturbance itself. Originality/value: The results demonstrate the satellite attitude control using reaction wheel is enhanced by PD-AFC attitude controller.
\end{abstract}

Keyword: Active force control; Reaction wheel; Satellite attitude control 
